AperTO - Archivio Istituzionale Open Access dell'Università di Torino

\title{
Design and simulation of an optimized e-linac based neutron source for BNCT research
}

\section{This is the author's manuscript}

Original Citation:

Availability:

This version is available http://hdl.handle.net/2318/1560702

since 2016-05-03T16:58:22Z

Published version:

DOI:10.1016/j.apradiso.2015.07.039

Terms of use:

Open Access

Anyone can freely access the full text of works made available as "Open Access". Works made available under a Creative Commons license can be used according to the terms and conditions of said license. Use of all other works requires consent of the right holder (author or publisher) if not exempted from copyright protection by the applicable law. 


\section{(2) \\ UNIVERSITÀ DEGLI STUDI DI TORINO}

This is an author version of the contribution published on:

Questa è la versione dell'autore dell'opera:

Applied Radiation and Isotopes, Vol. 106, 2015

DOI: $\underline{10.1016 / j . a p r a d i s o .2015 .07 .039}$

The definitive version is available at:

La versione definitiva è disponibile alla $U R L$ :

http://www.sciencedirect.com/science/article/pii/S0969804315301214 


\title{
Design and simulation of an optimized e-Linac based neutron source for BNCT research
}

\author{
E. Durisi ${ }^{1,2}$, K. Alikaniotis ${ }^{1}$, O. Borla ${ }^{5}$, F. Bragato ${ }^{3}$, M. Costa $^{1,2}$, G. Giannini $^{3,4}$, V. Monti $^{1}$, \\ L. Visca ${ }^{1,2}$, G. Vivaldo ${ }^{2}$, A. Zanini ${ }^{2}$ \\ ${ }^{1}$ Dipartimento di Fisica, Università di Torino, Via P. Giuria 1, 10125 Torino, Italy \\ ${ }^{2}$ Istituto Nazionale di Fisica Nucleare Sez. di Torino, Via P. Giuria 1, 10125 Torino, Italy \\ ${ }^{3}$ Università di Trieste, Via Valerio 2, 34127 Trieste, Italy \\ ${ }^{4}$ Istituto Nazionale di Fisica Nucleare Sez. di Trieste, Via Valerio 2, 34127 Trieste, Italy \\ ${ }^{5}$ Politecnico di Torino, Corso Duca degli Abruzzi 24, 10129 Torino, Italy
}

\begin{abstract}
The paper is focused on the study of a novel photo-neutron source for BNCT preclinical research based on medical electron Linacs. Previous studies by the authors already demonstrated the possibility to obtain a mixed thermal and epithermal neutron flux of the order of $10^{7} \mathrm{~cm}^{-2} \mathrm{~s}^{-1}$. This paper investigates possible Linac's modifications and a new photo-converter design to rise the neutron flux above $510^{7} \mathrm{~cm}^{-2} \mathrm{~s}^{-1}$, also reducing the gamma contamination.
\end{abstract}

\section{Introduction}

At present, Boron Neutron Capture Therapy preclinical research that includes the development of more efficient boron carriers, the study on cell specimens of tumor pathologies more appropriate to be treated with BNCT, as well as, new methods for dosimetry, would much profit of easy accessible and low cost neutron sources. So far, this research is being performed mainly at nuclear reactors with restricted access protocols and high costs of operation and maintenance. In the last years, in the BNCT scientific community the interest on accelerator based facilities has significantly increased (Esposito et al., 2009). These facilities are mainly based on light charged particle beams (proton or deuteron) on suitable targets, e.g. ${ }^{7} \mathrm{Li},{ }^{9} \mathrm{Be}$, deuterium and tritium. Considering the neutron production rate and the energy distribution, one of the most promising technique turned out to be the combination of proton beam with lithium (Culbertson et al., 2004). However, efficient target cooling systems are still under development to increase the proton current and consequently the neutron flux. In the last ten years, compact neutron generators based on D-D or D-T fusion reactions were also investigated, but attempts to produce high intensity fluxes have been relatively unsuccessful (IAEA, 2000).

Since 2007, projects funded by the "Istituto Nazionale di Fisica Nucleare" (INFN) and the Italian Ministry of Education, University and Research (MIUR), have investigated the possibility of a mixed thermal and epithermal neutron source based on photo-disintegration process $(\gamma, n)$. The bremsstrahlung photon beam, coming out from a high energy medical e-Linac (18-25 MV) hits a suitable self-shielded photo-converter. Primary neutrons with energy between $700 \mathrm{keV}-1 \mathrm{MeV}$ can be produced via Giant Dipole Resonance (GDR) reaction: this process is characterized by a cross section linearly dependent on the target material $(Z)$ and it can occur only when the gamma energy is above a certain threshold. Commonly used high $\mathrm{Z}$ elements, such as $\mathrm{Pb}$ and $\mathrm{W}$, have energy threshold of $7.41 \mathrm{MeV}$ and $7.42 \mathrm{MeV}$ respectively. Then, primary neutrons need to be moderated to lower energy as required for BNCT. Low Z materials, such as $\mathrm{D}_{2} \mathrm{O}$ and $\mathrm{C}_{2} \mathrm{H}_{4}$, are more suitable for this purpose. In this framework, a small photo-converter prototype "Phones Bianco" has been designed and manufactured to obtain a mixed thermal and epithermal neutron field. In the following, we will refer to such a field with the name "hyperthermal" as used in (Gonzalez et al., 2004). Several measurements have been carried out in different hospitals and the hyperthermal neutron field has been characterized with bubble neutron detectors, $\mathrm{Gd}_{2} \mathrm{O}_{3}$ gadolinium imaging plates (Bevilaqua et al., 2007) and microstrip silicon detectors (Mattera et al., 2009). Its use with biological specimens has also been demonstrated, although an irradiation time of three hours has been necessary to reach the fluence of $10^{11} \mathrm{~cm}^{-2}$ at the sample position (Borasio et al., 2008). 
This paper proposes possible Linac modifications and a novel photo-converter design in order to increase the hyperthermal neutron flux limiting the undesired contaminations.

\section{Material and Methods}

\subsection{Monte Carlo simulations}

The photo-converter simulation studies have been carried out using MCNP4B-GN code (NEA$1733)$ an extended version of MCNP4B (MCNP4B, 1997) to take into account the $(\gamma, n)$ processes in high $\mathrm{Z}$ and low $\mathrm{Z}$ elements for photons up to $30 \mathrm{MeV}$. Therefore, MCNP4B-GN has been used to treat the electromagnetic cascade and the photo-neutron production and transport. The employed photo-reaction model assumes that the dominant neutron emission mechanism is evaporation, with a maxwellian neutron energy distribution and an isotropic angular distribution. A small direct neutron emission component, with resulting $\mathrm{E}_{\mathrm{n}}>2 \mathrm{MeV}$, is also considered (Ongaro et al. 1999; Zanini et al., 2004).

To check the model validity a comparison with MCNP5, which uses different cross sections libraries, has been done. No significant effects have been observed.

In the Monte Carlo the photo-neutron production is simulated referring to the accelerator electron beam current. The size of each run has been dimensioned in order to have a statistical uncertainty below 5\% (that represents one standard deviation). To deal with the low GDR photo-neutron production cross section with respect to the total one (about two orders of magnitude), specific variance reduction techniques have been employed.

\subsection{The photo-converter design}

The role of the photo-converter is to produce neutrons by $(\gamma, n)$ reaction and to moderate these down to hyperthermal energy range $(<10 \mathrm{keV})$, minimizing gamma and fast neutron contamination. In particular, the gamma background is due both to primary unconverted photons (at low energy) and to those coming from neutron capture processes in the moderator (e.g. photons in the MeV range coming from Carbon and Hydrogen capture reaction).

The "Phones Bianco" prototype, mentioned above, has a simple geometry shown in figure 1 containing mainly $10 \mathrm{~cm}$ thick lead neutron production core followed by heavy water moderator and surrounded by graphite reflector blocks. The entire apparatus is covered by a $1 \mathrm{~cm}$ thick polyethylene layer.

Lead photo-neutron cross section shows a GDR peak of 600 mbarn around $13 \mathrm{MeV}$. The convolution of this peak with the bremsstrahlung spectrum gives the theoretical amount of convertible photons. Emitted neutrons have an energy spectrum characterized by a mean energy around $700 \mathrm{keV}-1 \mathrm{MeV}$ and they can be reduced to hyperthermal range mainly through elastic scattering in moderator materials. Heavy water, contained in carbon fiber boxes, has been chosen for its high moderation power combined with its low neutron capture cross section.

A cavity of $20 \mathrm{~cm} \times 20 \mathrm{~cm} \times 5 \mathrm{~cm}$, suitable to host samples to be irradiated, is placed in the inner part of the photo-converter. In order to increase the hyperthermal neutron flux inside the cavity a closed configuration has been chosen. The structure closing the cavity is made of heavy water and polyethylene in order to scatter neutrons back to the cavity.

New geometries have also been investigated to optimize the neutron production and to reduce the capture loss. Figure 2 shows, as an example, a cross section of the "Montezuma" photo-converter scheme. It presents a core made of a pyramidal structure of lead blocks separated by low Z material layers. This design offers a larger impact surface for photons at the production target, which contributes to increase the primary neutron flux without loosing moderation efficiency. 

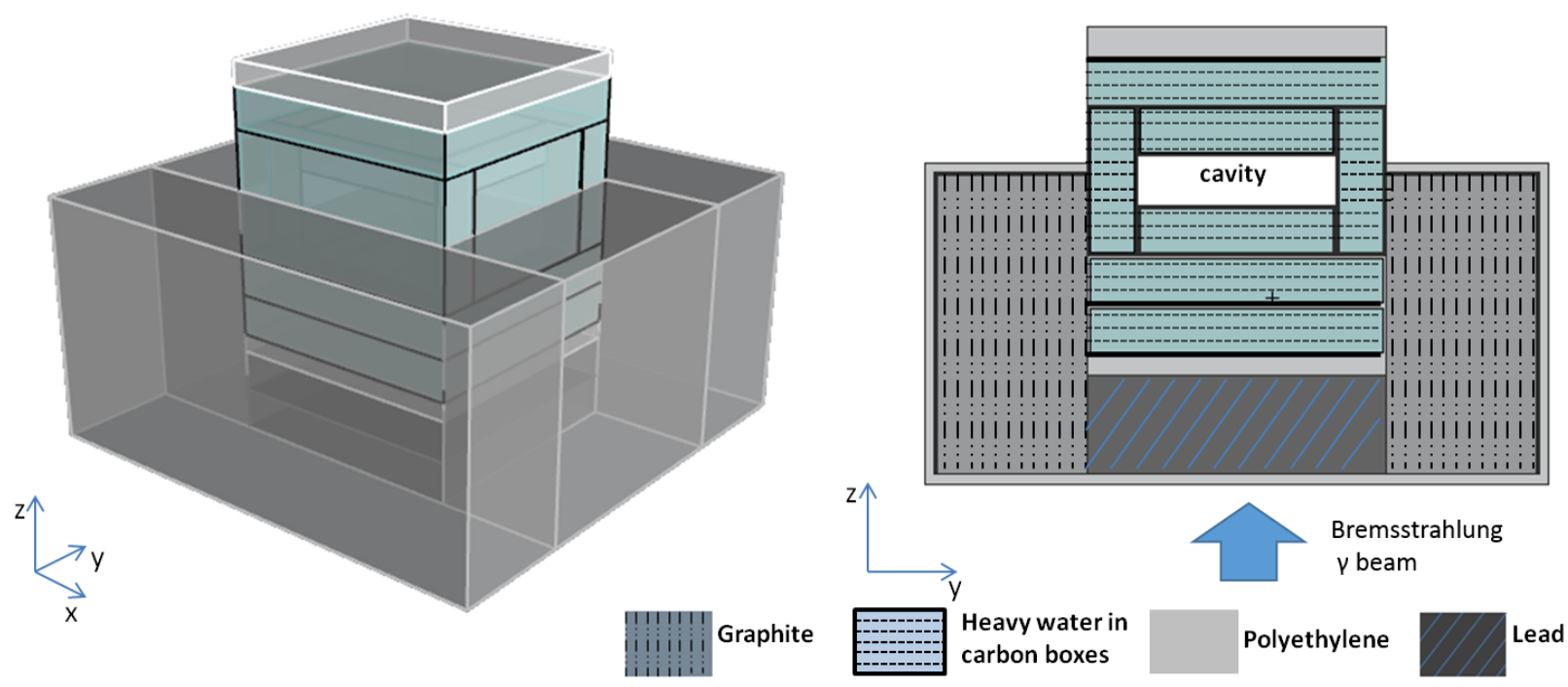

Fig. 1: “Phones Bianco" photo-converter assembly (bremsstrahlung photons are along z-axis).

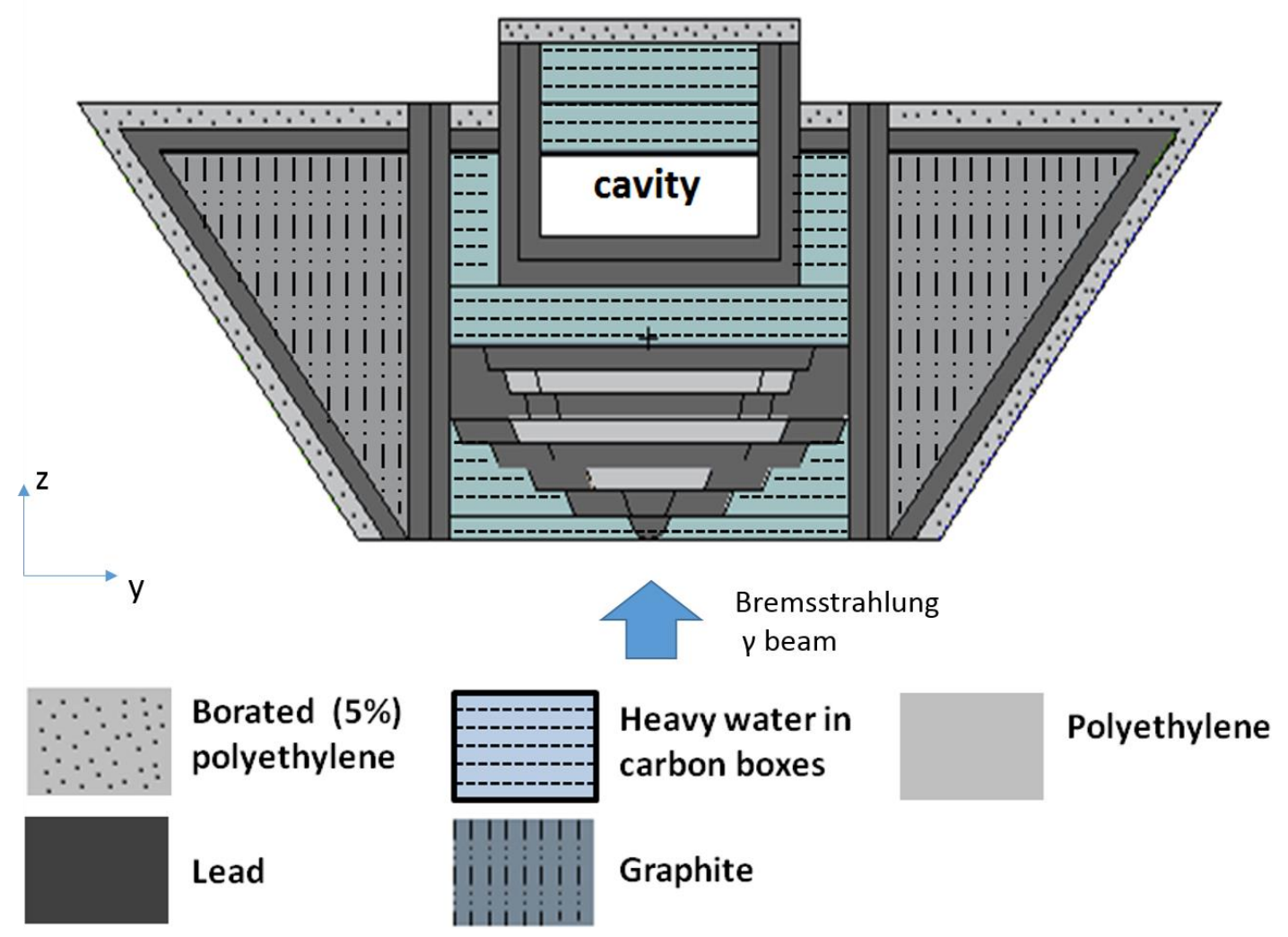

Fig. 2: "Montezuma" photo-converter assembly (bremsstrahlung photons are along z-axis).

\subsection{The Elekta Precise 18 MV e Linac}

Since the final neutron beam is strictly related to the photon spectrum impinging on the photoconverter, the main components of an Elekta Precise $18 \mathrm{MV}$ e-Linac have been included in the MCNP4B-GN input file.

In order to enhance the neutron fluence rate, several simulations have been carried out considering, 
in agreement with the Elekta technical division, possible modifications in the structure of the accelerator head and in its working parameters.

First of all, the removal of the flattening filter has been investigated. This is a stainless-steel cone normally used to make the photon field homogeneous in energy and space at the patient plane. Its removal brings a modification in the photon spectrum impinging on the photo-converter core, allowing a higher photo-neutron production.

Then, the increase of the primary electron beam energy has also been considered. This would rise the mean energy of the photon spectrum and consequently the neutron production. Within this respect, the Elekta technical division agreed to increase the electron energy up to $20 \mathrm{MeV}$ from the nominal $18 \mathrm{MeV}$.

Finally, the modifications of the duty cycle parameters (nominal current $I$, pulse duration $T$ and repetition rate $v$ ) have been investigated. Elekta's technicians indicated that the nominal current $I$ could be rised from $35 \mathrm{~mA}$ to $50 \mathrm{~mA}$, the pulse duration $T$ from $2.4 \mu$ s to $2.8 \mu$ s and the repetition rate $v$ could be set to $200 \mathrm{~Hz}$. With these combined actions an electron rate $\mathrm{N}_{\mathrm{e}}$ (defined as $N_{e}=6.25$ $10^{18} I T \mathrm{v}$ ) equal to $1.7510^{14} \mathrm{~s}^{-1}$ at the target position can be obtained, instead of the nominal value of $1.0510^{14} \mathrm{~s}^{-1}$.

\section{Results}

This section summarizes the main results obtained with MCNP4B-GN simulations of the Elekta Precise 18 MV e_Linac in different configurations, coupled with "Phones Bianco" and "Montezuma" photo-converters.

Figure 3 shows the mean photon energy spectrum, calculated on a $(20 \times 20) \mathrm{cm}^{2}$ surface at $50 \mathrm{~cm}$ from the Linac target, in different machine configurations. By removing the flattening filter in the Linac head and considering only photons with energy above the photo-neutron production threshold $(>7.41 \mathrm{MeV}$ for lead) an increment of a factor 2.2 can be obtained. Moreover, the increase of the primary electron energy up to $20 \mathrm{MeV}$ brings a further 1.44 gain factor in the "useful" photon flux.

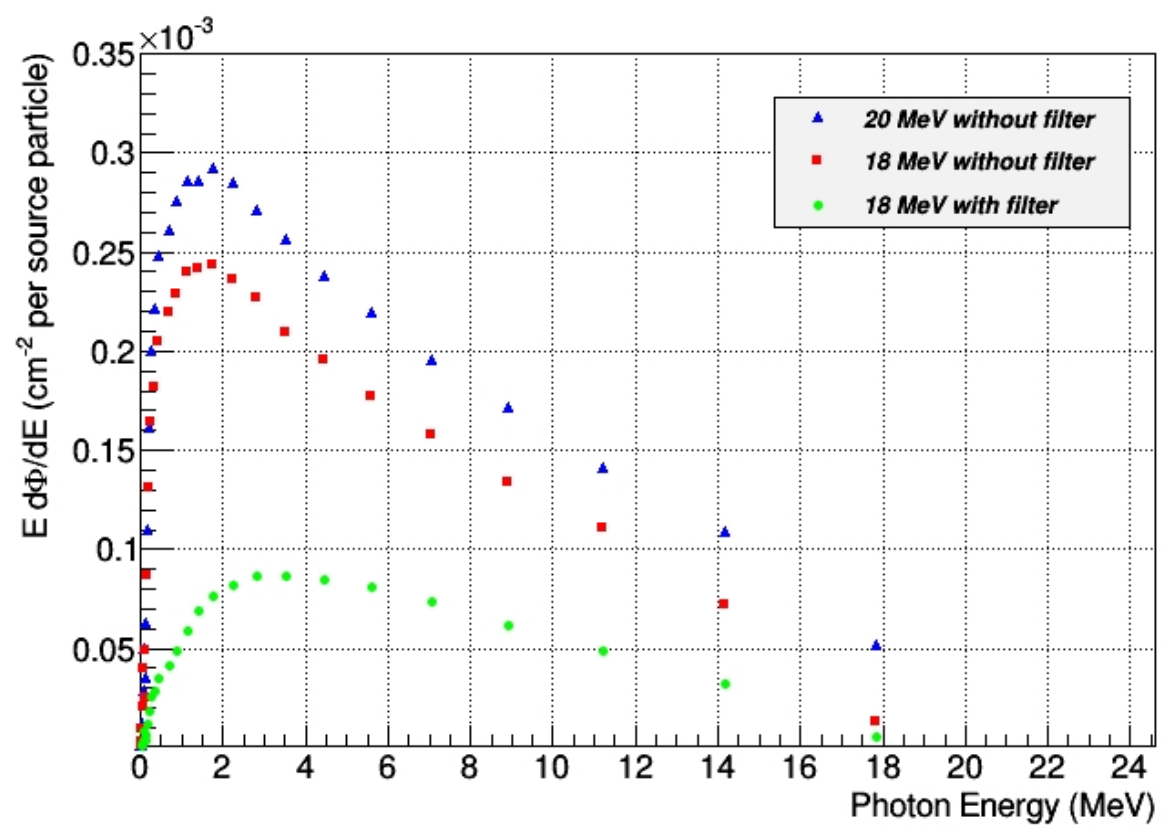

Fig. 3: Photon spectra calculated on a $(20 \times 20) \mathrm{cm}^{2}$ surface with MCNP4B-GN at $50 \mathrm{~cm}$ from the target. The lowest curve corresponds to the nominal accelerator configuration; the intermediate one is obtained removing the flattening filter from the Linac head; the highest curve is obtained removing the flattening filter and rising the electron energy up to $20 \mathrm{MeV}$.

As far as the neutron production and moderation is concerned, figure 4 shows the mean neutron 
energy spectra calculated in the cavity coupling "Phones Bianco" photo-converter with the Elekta Precise $18 \mathrm{MV}$ e-Linac in the configurations above described. In standard conditions (green circle) the hyperthermal neutron fluence amounts to $9.3510^{-8} \mathrm{~cm}^{-2}$ per source particle, corresponding to a neutron flux in the cavity of $9.8310^{6} \mathrm{~cm}^{-2} \mathrm{~s}^{-1}$ using $\mathrm{N}_{\mathrm{e}}=1.0510^{14}$ electrons per second. The thermal $(<0.4 \mathrm{eV})$ component accounts for $75 \%$, while the epithermal $(0.4 \mathrm{eV}<\mathrm{E}<10 \mathrm{keV})$ and the fast $(>10$ $\mathrm{keV}$ ) ones are of the order of $19 \%$ and $6 \%$ respectively. By removing the flattening filter (red square) an increment of a factor 2.1 in the hyperthermal flux is observed with minor effects on the percentage of the unwanted fast component. The best configuration is obtained removing the filter and increasing the electron energy and the accelerator duty cycle (blue triangle): a hyperthermal neutron flux of $5.1910^{7} \mathrm{~cm}^{-2} \mathrm{~s}^{-1}$ (using $\mathrm{N}_{\mathrm{e}}=1.7510^{14}$ electrons per second) is obtained.

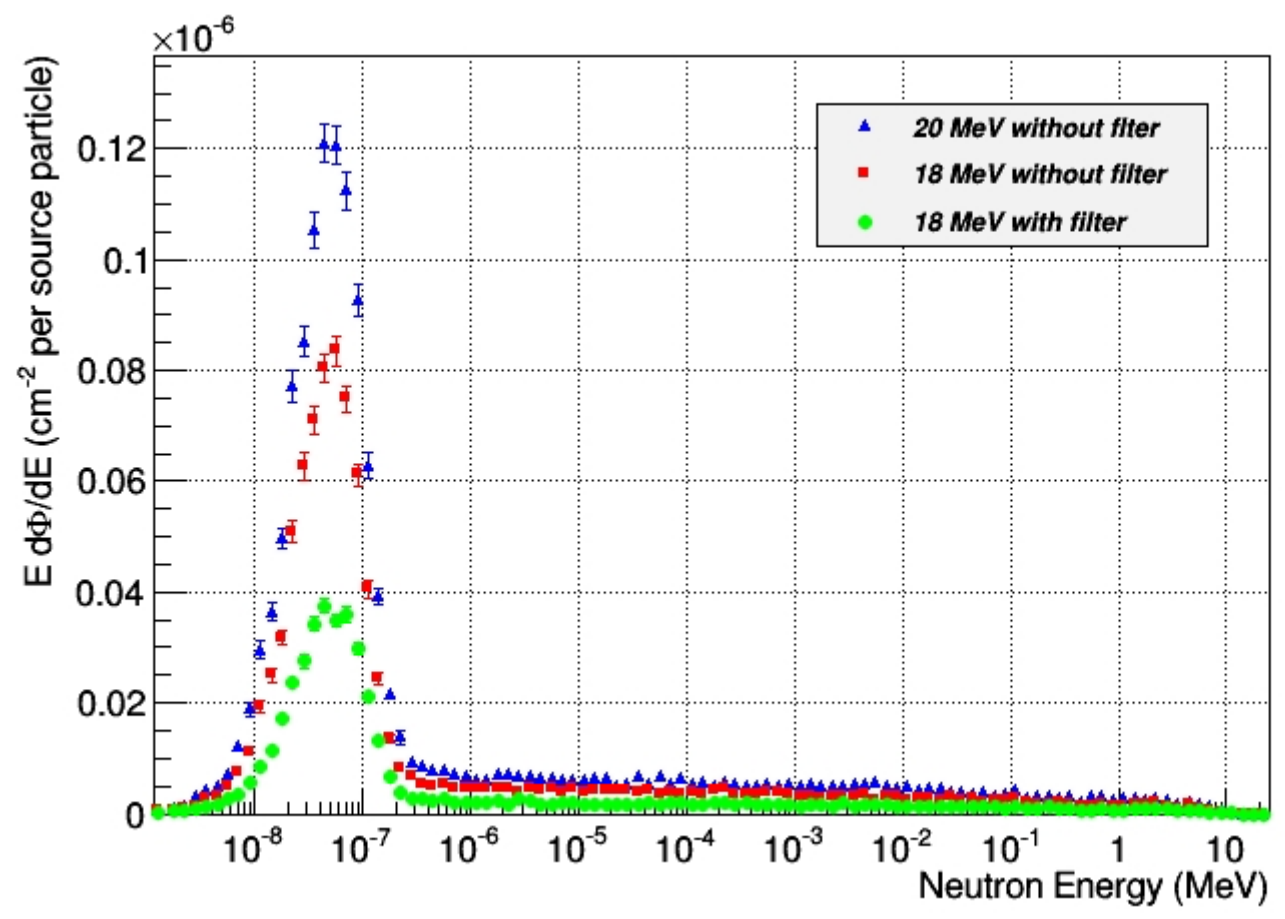

Fig. 4: Mean neutron spectra calculated with MCNP4B-GN inside the irradiation cavity of "Phones Bianco" using different Linac configurations.

Using the "Montezuma" photo-converter scheme and the optimized e-Linac configuration two main effects have been observed inside the cavity. The hyperthermal neutron flux increases up to (5.89 \pm $0.05) 10^{7} \mathrm{~cm}^{-2} \mathrm{~s}^{-1}$, as indicates in table 1 , while the gamma contamination, shown in figure 5 , is reduced by a factor 2.5 . The low energy gammas in the cavity are removed together with a sensitive reduction of about $30 \%$ of gamma above $1 \mathrm{MeV}$.

However, the "Montezuma" photo-converter shows a higher fast neutron contamination with respect to the "Phones Bianco" scheme. Therefore, further studies and simulations devoted to the test new materials and new shaping assemblies are under investigation. 


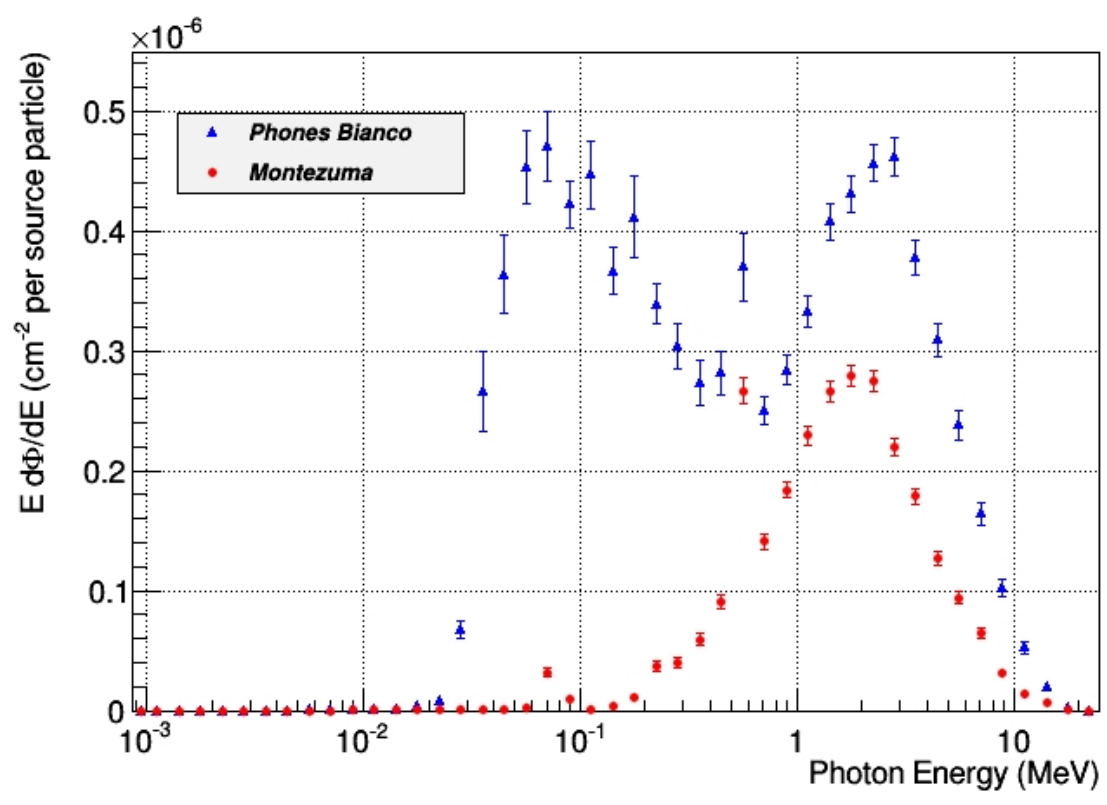

Fig 5: Mean photon spectra calculated with MCNP4B-GN inside the irradiation cavity. Comparison between "Phones Bianco" and "Montezuma" photo-converters on the same optimized Linac configuration.

\begin{tabular}{|c|c|c|c|c|c|}
\hline $\begin{array}{l}\text { Elekta Precise } \\
\text { with "Phones } \\
\text { Bianco" }\end{array}$ & $\begin{array}{l}\text { Hyperhermal } \\
\text { neutron fluence per } \\
\text { source particle } \\
\left(\mathrm{cm}^{-2}\right)\end{array}$ & $\begin{array}{l}\text { Electron rate } \\
\mathrm{N}_{\mathrm{e}}\left(\mathrm{s}^{-1}\right)\end{array}$ & $\begin{array}{l}\text { Hyperhermal } \\
\text { neutron flux } \\
\left(\mathrm{cm}^{-2} \mathrm{~s}^{-1}\right)\end{array}$ & $\begin{array}{l}\text { Gamma } \\
\text { contamination } \\
\mathbf{D}_{\boldsymbol{\gamma}} / \boldsymbol{\phi} \text { hyperthermal } \\
\left(\mathbf{G y} \mathbf{~ c m}^{\mathbf{2}}\right) \\
\end{array}$ & 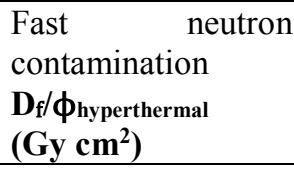 \\
\hline $\begin{array}{l}18 \mathrm{MeV} \text { with } \\
\text { filter }\end{array}$ & $(9.35 \pm 0.18) 10^{-8}$ & $1.0510^{14}$ & $(9.83 \pm 0.19) 10^{6}$ & $(1.13 \pm 0.03) 10^{-10}$ & $(8.6 \pm 0.5) 10^{-13}$ \\
\hline $\begin{array}{ll}18 & \mathrm{MeV} \\
\text { without filter }\end{array}$ & (1.99 0.03$) 10^{-7}$ & $1.0510^{14}$ & $(2.09 \pm 0.03) 10^{7}$ & (1.64 0.03$) 10^{-10}$ & $(8.7 \pm 0.3) 10^{-13}$ \\
\hline $\begin{array}{ll}20 & \mathrm{MeV} \\
\text { without filter }\end{array}$ & 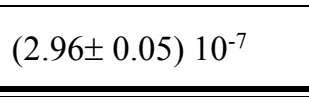 & $1.7510^{14}$ & $(5.19 \pm 0.08) 10^{7}$ & $(1.35 \pm 0.02) 10^{-10}$ & $(1.00 \pm 0.04) 10^{-12}$ \\
\hline \multicolumn{6}{|l|}{$\begin{array}{l}\text { Elekta Precise } \\
\text { with } \\
\text { "Montezuma" }\end{array}$} \\
\hline $\begin{array}{ll}20 & \mathrm{MeV} \\
\text { without filter }\end{array}$ & (3.36 0.03) $10^{-7}$ & $1.7510^{14}$ & $(5.89 \pm 0.05) 10^{7}$ & (5.07士 0.09) $10^{-11}$ & $(3.2 \pm 0.1) 10^{-12}$ \\
\hline
\end{tabular}

Table 1. Summary of the main simulation results obtained coupling two photo-converters with the Elekta Precise 18MV e-Linac in different configurations.

\section{Conclusion}

At present, the BNCT scientific community is very interested in finding easy accessible and low cost neutron sources for preclinical and clinical studies.

This paper proposes to use a commercial medical e-Linac coupled with a dedicated self-shielded photo-converter to obtain a hyperthermal neutron field inside a small cavity. Using appropriate photo-converter geometries and materials, and modifying the machine working parameters together with the removal of the accelerator filters, a hyperthermal neutron flux as high as (5.89 \pm 0.05$) 10^{7}$ $\mathrm{cm}^{-2} \mathrm{~s}^{-1}$ has been achieved. This results in an improvement of the useful neutron flux of almost a factor six with respect to previous studies (Bevilaqua et al., 2007) and a correspondent reduction of 
the irradiation time. The gamma contamination in the cavity has also been reduced by almost a factor 2.5 while the fast neutron contamination should be still decreased.

The use of commercial e-Linac makes this approach easily reproducible and available to the scientific community.

\section{Acknowledgments}

The authors would like to acknowledge the INFN and the Italian Ministry MIUR for the economic support to the project.

\section{References}

Bevilacqua, R., et al., 2007. Phones: A novel approach to BNCT with conventional radiotherapy accelerators. Nuclear instrument and Methods in Physics Research A572, 231-232

Borasio, P., et al., 2008. A novel approach to the study of 10B uptake in human lung by ex-vivo BPA perfusion, proceeding of 13-ICNCT Florence, Italy

Culbertson, C.N., et al., 2004. In-phantom characterisation studies at the Birmingham AcceleratorGenerated epithermal Neutron Source (BAGINS) BNCT facility. Applied Radiation and Isotopes $61,733-738$

Esposito, J., et al., 2009. Be target development for the accelerator based SPES_BNCT facility at INFN Legnaro. Applied Radiation and Isotopes 67, S270-S273

Gonzalez, S.J., et al., 2004. First BNCT treatment of a skin melanoma in Argentina: dosimetric analysis and clinical outcome. Applied Radiation and Isotopes 61, 1101-1105

IAEA-TECDOC-1153, 2000. Use of accelerator based neutron source

Mattera, A., et al., 2009. Boron imaging with a microstrip silicon detector for applications in BNCT Nuclear instrument and Methods in Physics Research A604 82-85

MCNP4B 1997. MCNP ${ }^{\mathrm{TM}}$ - a general Monte Carlo n-particle transport code, version 4V LA12625-M Manual ed J F Briesmeister

Ongaro C., et al., 1999. Monte Carlo simulation of the photo-production in the high-Z components of radiotherapy linear accelerators Monte Carlo Methods and Applications 5, 69-80

Zanini A., et al., 2004. Monte Carlo simulation of the photo-neutron field in linac radiotherapy treatments with different collimation systems Physics in Medicine and Biology 49, 1-12 